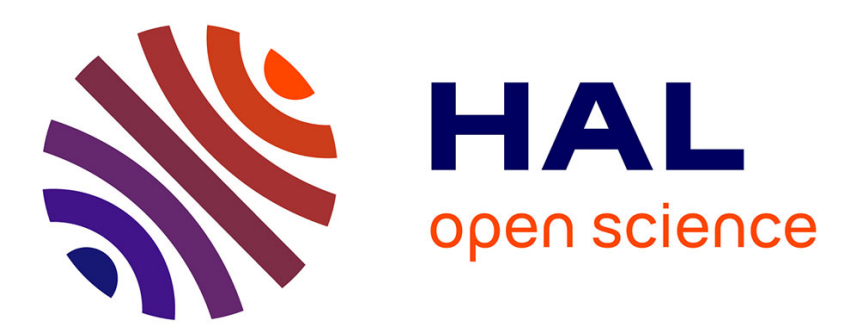

\title{
Restoration of 3D medical images with total variation scheme on wavelet domains (TVW)
}

\author{
Arnaud Ogier, Pierre Hellier, Christian Barillot
}

\section{To cite this version:}

Arnaud Ogier, Pierre Hellier, Christian Barillot. Restoration of 3D medical images with total variation scheme on wavelet domains (TVW). SPIE Medical Imaging: Image Processing, Feb 2006, San Diego, CA, pp.465-473. inria-00001159

\section{HAL Id: inria-00001159 \\ https://hal.inria.fr/inria-00001159}

Submitted on 22 Mar 2006

HAL is a multi-disciplinary open access archive for the deposit and dissemination of scientific research documents, whether they are published or not. The documents may come from teaching and research institutions in France or abroad, or from public or private research centers.
L'archive ouverte pluridisciplinaire HAL, est destinée au dépôt et à la diffusion de documents scientifiques de niveau recherche, publiés ou non, émanant des établissements d'enseignement et de recherche français ou étrangers, des laboratoires publics ou privés. 


\title{
Restoration of 3D medical images with total variation scheme on wavelet domains (TVW).
}

\author{
Arnaud Ogier, Pierre Hellier and Christian Barillot \\ Unit/Project Visages U746, INSERM-INRIA-CNRS-Univ-Rennes1, Campus de Beaulieu, 35042 Rennes Cx, France
}

\begin{abstract}
The multiplicity of sensors used in medical imaging leads to different noises. Non informative noise can damage the image interpretation process and the performance of automatic analysis. The method proposed in this paper allows compensating highly noisy image data from non informative noise without sophisticated modeling of the noise statistics. This generic approach uses jointly a wavelet decomposition scheme [3] and a non-isotropic Total Variation filtering [9] of the transform coefficients. This framework benefits from both the hierarchical capabilities of the wavelet transform and the well-posed regularization scheme of the Total Variation. This algorithm has been tested and validated on test-bed data, as well as different clinical MR and 3D ultrasound images, enhancing the capabilities of the proposed method to cope with different noise models.
\end{abstract}

keywords: Denoising, wavelets, total variation, restoration, ultrasound.

\section{Introduction}

New applications of medical imaging systems are parallel to the development or the evolution of new machinery which comes with specific artifacts that are still only partially corrected. This is the case for instance with high field MRI, 3D ultrasound imaging or other modalities. With regards to the images to process and analyze, these artifacts translate into additive or multiplicative noise, and geometric or intensity distortions that drastically affect not only the visual interpretation, but also most of the segmentation or registration algorithms, and the quantitative measures that follow. Ideally, a better comprehension of these artifacts would necessitate an increased dialog between the physicists (who make the images), the computer scientists (who process the images) and the clinicians (who interpret the images). In practice building such a realistic model, well dedicated to each image formation stage, leads to specific methods and evenmore are often hard to define due to the high complexity of the image formation workflow. This should lead to define new, specifically-designed algorithms, based on mathematical modeling of image formation while keeping this modeling as generic as possible. As an example, for the concern of 3D ultrasound images, or even high field MRI $(>=3 T)$, partially because of the challenging aspect of this problem especially in the clinical context, very few restoration schemes have been proposed. It is clear that only an approach balancing between the mathematical modeling of the image formation and a relative genericity of the method could give an adequate response to this problem.

The main contribution of the paper is to propose a generic framework based on wavelets and total variation minimization to restore images. We show that this restoration scheme outperforms the TV restoration scheme and optimal wavelet threshold restoration. 


\section{Context}

Generally, the observed image $f$ can be modeled as a degraded version of the noiseless image $u$, as :

$$
f=h * u+v
$$

where $v$ is an additive white noise and $h$ is considered as a low pass filter. These notations will be used through out the paper.

Restoration methods can be broadly classified in three groups: stochastic modeling based on Markov random field theory $[4,11]$, with the maximization of a MAP criteria; wavelet theory based on decomposition techniques $[5,7,8,10]$; and projection into the wavelet domain; and variational methods $[6,9]$, based on the resolution of partial differential equation.

Images are considered continuous and defined in the $L^{2}$ space, (space of square integrable functions). Nevertheless, "real-life" images have discontinuities when crossing edges. In order to take into account this fact, the Bounded Variation framework has been introduced in image analysis, and is detailed in the next section.

\subsection{Introduction of the Total Variation model}

Rudin et al. [9] noted that images are better defined in the space of Bounded Variation $B V$. The $B V$ norm allows discontinuities on curves and hence preserved edges. To allow discontinuities in images, the derivative $D u$ of $\mathrm{u}$, can be represented as a distribution and is defined as a Radon measure. If $u \in \mathcal{C}^{1}$, then we can approximate the term $\int|D u|$ by $\int|\nabla u|$. For simplicity, Rudin et al. propose to decompose the whole image as:

$$
f=u_{\epsilon_{B V}}+v_{\epsilon_{L^{2}}}
$$

where $f$ is the observed image (the $\mathrm{h}$ filter is consider equivalent to unicity in the following framework), $u$ is the noiseless image (contains the geometrical information) and $v$ is the noise.

Solving this problem leads to the minimization of the following expression:

$$
\hat{u}=\arg \min _{u} \int|\nabla u|+\lambda \int\|f-u\|_{2}^{2}
$$

where $\lambda$ is the Lagrange multiplier.

\subsection{Wavelets method for image denoising.}

The basic idea of wavelets is to analyze a signal at different scales. A wavelet is a function $\Psi \in L_{2}$ such that, $\int_{\infty}^{\infty} \Psi(x) d x=0 . \Psi(x)$ is called mother wavelet. It is centered in the neighborhood of $x=0$ and has a unit norm, i.e $\|\Psi(x)\|=1$

The mother wavelet entails the wavelets family $\Psi_{a, b}(x), a>0, b \in \mathcal{R}$ by a scaling factor $(a)$ and a translation $(b)$.

$$
\Psi_{a, b}(x)=\frac{1}{\sqrt{a}} \Psi\left(\frac{x-b}{a}\right)
$$

The discrete wavelet transformation (DWT) introduced by Mallat [7] decomposes the image in a multiresolution manner, providing at each resolution level one low resolution approximation and seven spatially oriented wavelet details (in 3D). It can be generalized to any positive dimension. In three dimensions (i.e. volumes), the filter banks are successively applied in the three directions. Wavelets coefficients that are supposed to be related to noise are then removed by two different methods [3]: hard thresholding (keep or kill), or soft thresholding (shrink or kill). In both cases, the coefficients that are below a given threshold are set to zero. The optimal threshold is $T=$ $\sigma \sqrt{2 \log N}$ in case of an additive Gaussian noise [3], where $N$ is the number of voxels in the highest frequency wavelet sub-band $H H H^{1}$ and $\sigma=\frac{M A D(H H H)}{0.675}$, where $M A D$ is the median absolute deviation.

\footnotetext{
${ }^{1}$ Wavelets decomposition in 3D leads to one low frequency and seven high frequencies.
} 


\section{TVW restoration based on wavelets and total variation}

We propose a method where the wavelet decomposition and TV are coupled. It is expected that this framework should beneficiate from the hierarchical decomposition capabilities of the wavelets and the well-posed regularization scheme of the TV. In practice, the image will be decomposed with wavelets and the resulting coefficient will be regularized using the total variation scheme. In Discrete Wavelet Transform (DWT), only the low frequency is decomposed. This turns out to be efficient for regular signals which information is essentially in the low frequencies. However, medical images are more complex and usefull information is to be found in the other band of frequencies. A decomposition with a 3D separable wavelet-packet transform is applied where all sub-bands are decomposed. It is assumed that the wavelet coefficients are composed of an additive noise and a real signal. After convergence, filtered coefficients are gathered so as to reconstruct the image.

$$
\mathcal{W}_{f_{(n, l)}}=\mathcal{W}_{u_{(n, l)}}+\mathcal{W}_{v_{(n, l)}}
$$

with $\mathcal{W}_{f_{(n, l)}}$ being the $n^{\text {th }}$ sub-band at the $l^{\text {th }}$ level of decomposition noised wavelet coefficients and $\mathcal{W}_{u_{(n, l)}}$ being the coefficients that contain the geometric information and $\mathcal{W}_{v_{(n, l)}}$ the noise.

We are inside a bounded space (Besov's space) where the TV algorithm can be used. For the $l^{\text {th }}$ level of decomposition, we get:

$$
\bigcup_{n=1}^{2^{l^{2}+2}} \mathcal{W}_{(n, l)}^{-1}\left[\min _{\mathcal{W}_{u_{(n, l)}}} \int\left|\nabla \mathcal{W}_{u_{(n, l)}}\right|+\lambda \int\left\|\mathcal{W}_{f_{(n, l)}}-\mathcal{W}_{u_{(n, l)}}\right\|_{2}^{2}\right]
$$

The minimization problem of a given energy term $J(u)$ for a functional $F$ defined in 3D can be written as:

$$
J(u)=\iint F\left(x, y, z, u, u_{x}, u_{y}, u_{z}\right) d x d y d z,
$$

This can be solved by using Euler-Lagrange:

$$
F_{u}-\frac{\partial}{\partial x} F_{u_{x}}-\frac{\partial}{\partial y} F_{u_{y}}-\frac{\partial}{\partial z} F_{u_{z}}=0
$$

where $u_{x}=\frac{\partial u}{\partial x}$. We minimize

$$
J(u)=\int|\nabla u|
$$

under the constraints

$$
\text { (1) } \int_{\Omega} u=\int f ; \quad(2) \int_{\Omega} \frac{1}{2}(f-u)^{2}=\sigma^{2} \text {. }
$$

The first constraint aims at preserving the mean value, while the second assumes that the noise is a white noise (zero mean and standard deviation $\sigma$ ).

The divergence term, resulting from Euler-Lagrange equation is :

$$
\operatorname{div}\left(\frac{\nabla u}{|\nabla u|}\right)=\frac{u_{x x}\left(u_{y}^{2}+u_{z}^{2}\right)+u_{y y}\left(u_{x}^{2}+u_{z}^{2}\right)+u_{z z}\left(u_{x}^{2}+u_{y}^{2}\right)}{\left(u_{x}^{2}+u_{y}^{2}+u_{z}^{2}\right)^{\frac{3}{2}}} \frac{-2\left(u_{x} u_{y} u_{x y}+u_{x} u_{z} u_{x z}+u_{y} u_{z} u_{y z}\right)}{\left(u_{x}^{2}+u_{y}^{2}+u_{z}^{2}\right)^{\frac{3}{2}}}
$$

The principal interest of this method allows to cope with different and complex noise naturally present in images. The Donoho et al. [3] threshold step is replaced by a fully automatic non linear process. The evaluation of Lagrange multiplier $\lambda$ in the resolution of total variation is often described as a fastidious and long calculus process, moreover, no significant amelioration has been proved in the literature by optimizing this parameter.

In practice $\lambda$ (positive and constant), is tuned by the user. Intuitively, $\lambda$ is chosen inversely 
proportional to the variance of the noise. The number of decomposition is in practice set to one. Increasing the number of decomposition leads to increase the computation time without notably increasing the quality of the images.

Little oscillations due to a finite number of coefficients (called Gibbs phenomena) can appear. The choice of the mother wavelet is important to limit this effect. In addition, the biorthogonal wavelet filter bank Cohen-Daubechies-Fauveau designed as a spline variant with less dissimilar length, [1] leads to reduce this problem.

\section{Results}

This restoration method was tested on various 3D images, ranging from 3T MRI to 3D ultrasound images, with validation on ground truth MRI volumes.

\subsection{Validation on MRI phantom data.}

Firstly, the evaluation of TVW method is performed on Brain web phantom ${ }^{2}$ [2]. This first experiment allows to study the performances of our method with respect to a ground truth. The evaluation is achieved with profiles comparison, visual assessment and a quantitative indexes computed from a classification procedure based on a Expectation Maximization (EM) method.

\subsection{1 a) Profiles comparison.}

In order to verify the localization preservation and the enhancement of the edges, a profile comparison (intensity distribution along a chosen line) is realized between the original image with $9 \%$ of noise $(f)$, denoised image $(\hat{u})$ and the ground truth $(u)$.

Results are presented in figure 1. Visually, the restored profile $(\hat{u})$ is close to the ground truth $(u)$. The localization of the edges is preserved and their dynamics increase slightly. On the flat areas, the restoration impact is especially efficient. As we can see in the restored profile, oscillations due to noise are significantly reduced.

\subsection{2 b) Visual assessment.}

The figure 2 shows the noisy image $f$, the restored image $\hat{u}$ and the difference image $(v=f-\hat{u})$. It appears that the restored image contains the useful geometric information. This is assessed by the difference image where the noise is dominant (very few informative structure are present in this image).

\subsection{3 c) Quantitative validation as a preprocess of segmentation task.}

The goal is to validate the proposed method w.r.t a segmentation task. An EM classifier was used, in order to compare the different restoration methods with respect to the same automatic classification process. Table 1 shows the classification results, comparing three methods: the optimal threshold on wavelets coefficients [3], the TV method [9] and our TVW method (Total Variation on Wavelets). Since ground truth is available, classification is assessed by computing quality measures such as overlap and Kappa indexes.

\subsection{Experiments on 3D clinical data.}

The TVW method was tested on real data. Experiments were carried out for two modalities where noise is important: 3T MR imaging and ultrasound imaging. These experiments are conducted in order to prove the independence of the TVW method with regards to the noise statistics, and the genericity of the proposed approach.

\footnotetext{
${ }^{2}$ http://www.bic.mni.mcgill.ca/brainweb/
} 

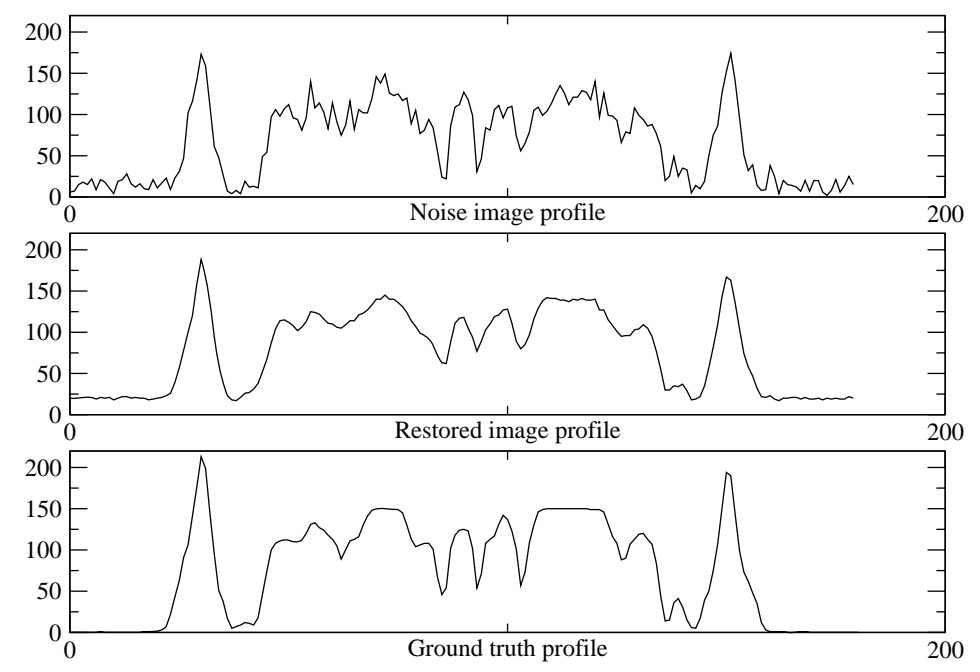

Figure 1: Profile of brain web MNI. Top: profile of the original image $f$. Center: profile of restored image $\hat{u}$. Bottom: profile of ground truth MNI $u$.
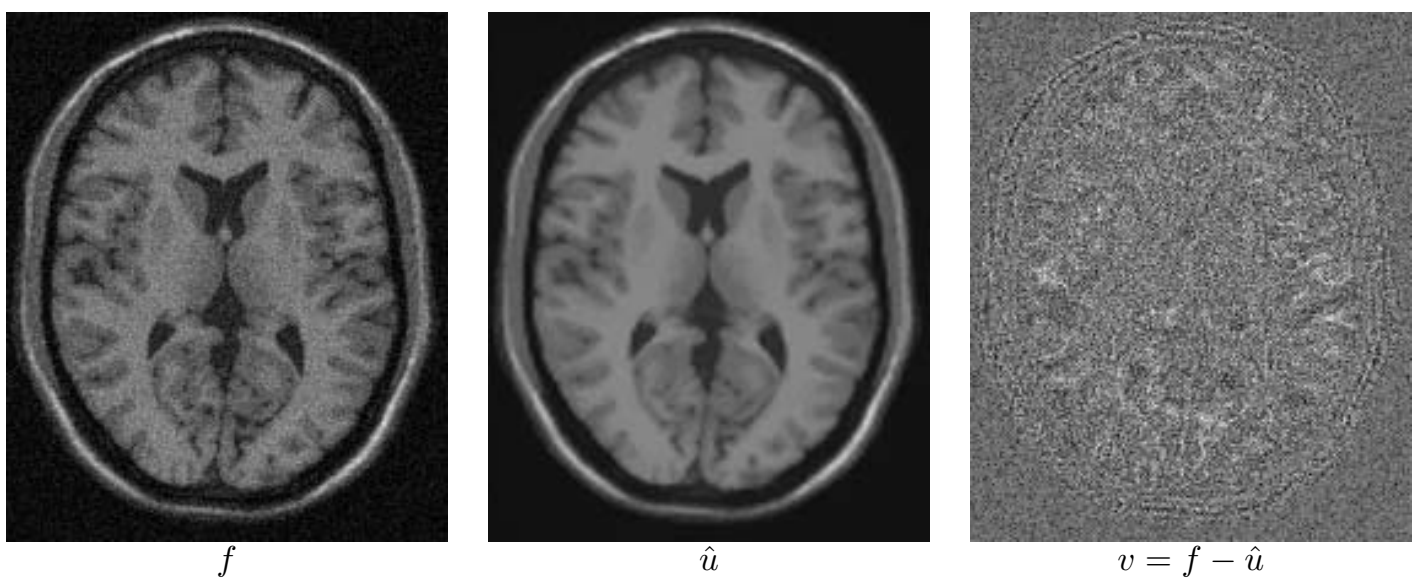

Figure 2: $f$ : initial noise image. $u$ : restored image. Right: difference image containing the noise.

\subsection{1 a) Experiment on 3T MRI.}

3T MRI is coming rapidly into the clinical practice. This allows a better image definition but at the expense of more pronounced noise. The restoration step is especially required for radiological interpretation task but also in order to perform image analysis which are always sensible to presence of noise. The figure 4 underlines the capability of TVW approach to edge conservation and white noise suppression. 


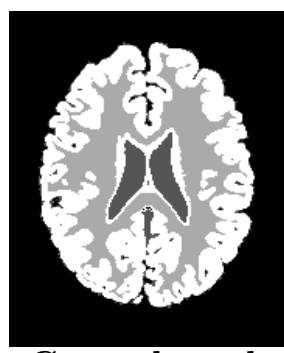

Ground truth

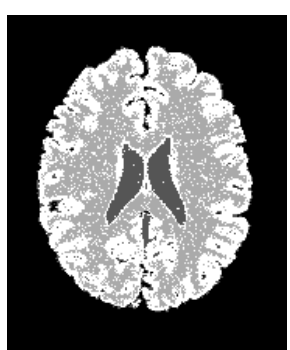

Noisy data

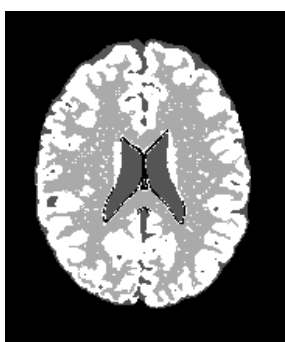

Threshold

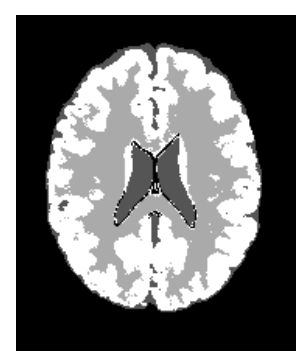

TV

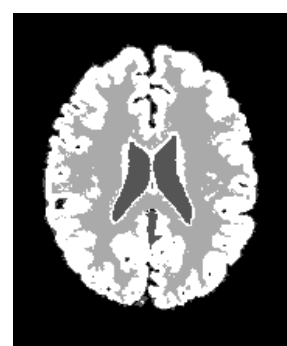

TVW

Figure 3: EM Classification in three classes. Left to right: The ground truth given by the MNI, result of EM segmentation on noisy data, result of EM segmentation after Donoho threshold process, result of EM segmentation after Rudin, Osher and Fatemi TV process, result of EM segmentation after our process.

\begin{tabular}{|c|cc|cc|}
\hline & $\begin{array}{c}\text { White Matter } \\
\text { Overlap }\end{array}$ & Kappa index & $\begin{array}{c}\text { Gray Matter } \\
\text { Overlap }\end{array}$ & Kappa index \\
\hline Noisy data $f$ & 0.49 & 0.66 & 0.74 & 0.85 \\
\hline Threshold data $\hat{u}_{T}$ & 0.66 & 0.80 & 0.83 & 0.91 \\
\hline TV data $\hat{u}_{T V}$ & 0.74 & 0.85 & 0.87 & 0.93 \\
\hline TVW data $\hat{u}_{T V W}$ & 0.78 & 0.88 & 0.89 & 0.95 \\
\hline
\end{tabular}

Table 1: Comparison between noisy data $9 \%$ of noise and different restoration methods for the same EM classification task. Only the restoration preprocessing differs between the experiments. This shows that proposed TVW method outperforms the other restoration method for a classification task.

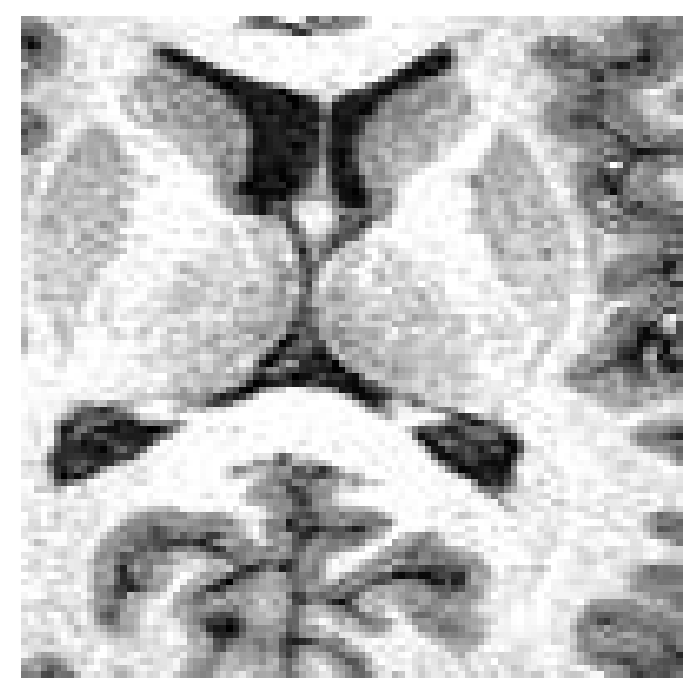

MRI

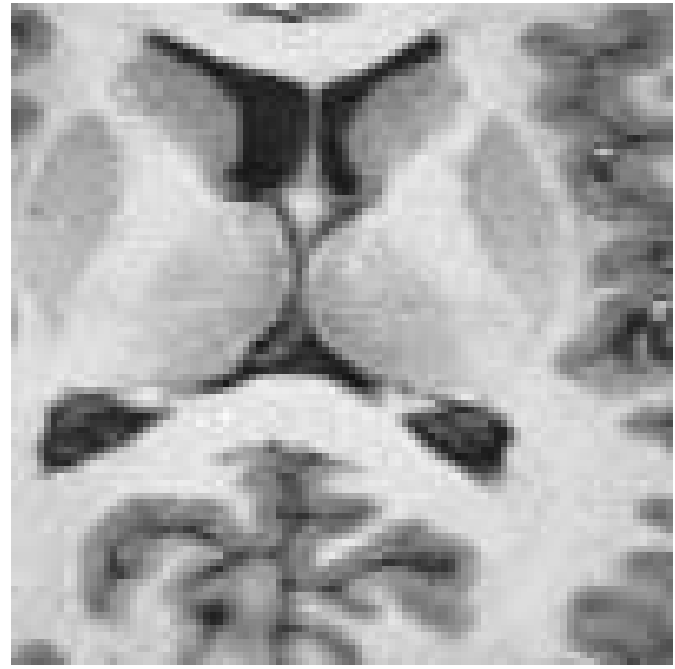

TVW Restoration

Figure 4: Results of 3T MRI restoration. The TVW methods reduces significantly the additive noise while preserving the edges.

\subsection{2 b) Experiments on 3D ultrasound images.}

The presence of multiplicative noise (speckle) in ultrasound images makes it difficult to apply image processing tools such as edge detection or image registration. The figure 5 presents the 
gradient magnitude of liver image before and after restoration achieved resp. with soft threshold, TV and TVW methods. These images are 2D slices taken from 3D volume, 3D restoration and 3D gradient computation. Visually, the soft optimal wavelet threshold restoration is not appropriate for speckle noise. The TVW and the TV methods obtain better results. The TVW is notably more efficient for noise suppression and edges preservation.

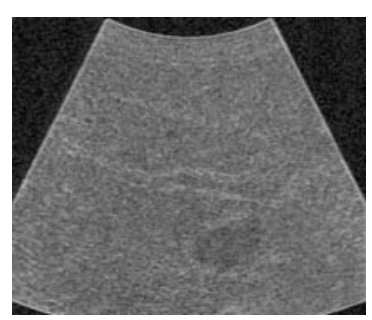

Liver

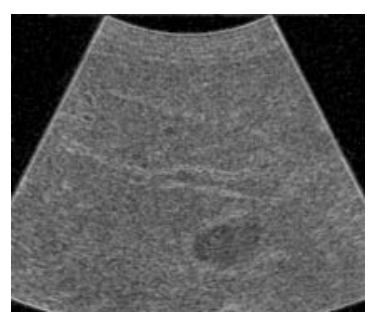

Soft threshold

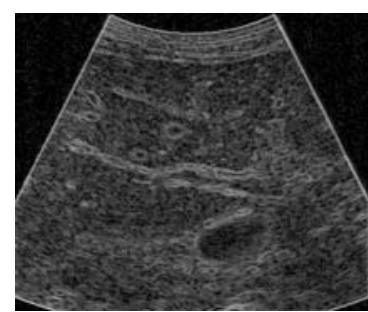

TV

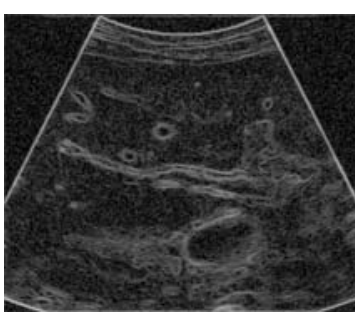

TVW

Figure 5: Gradient of liver US image. From left to right: gradients of initial image, optimal threshold, TV and TVW results. Thanks to its generic nature, the TVW improves the edge detection and reduces noise.

\section{Conclusion}

This paper presented a 3D restoration method using jointly a Total Variation framework and wavelets decomposition. This approach preserves edges, decreases noise and is generic w.r.t. the noise nature. Validation was performed on MRI test-bed data and enhance the capability of the Total Variation on Wavelets coefficients (TVW) to restore noisy data in order to improve the performance of a classification task. Experiments on clinical data sets were carried out on 3D MR and 3D ultrasound images. Similarly, these experiments have shown that TVW outperforms classical TV and wavelets approaches.

\section{References}

[1] A. Cohen, I. Daubechies, and J.C. Feauveau. Biorthogonal Bases of Compactly Supported Wavelets. Communications on Pure and Applied Mathematics, 45(5):485-560, May 1992.

[2] D.L. Collins, A.P. Zijdenbos, V. Kollokian, J.G. Sled, N.J. Kabani, C.J. Holmes, and A.C. Evans. Design and construction of a realistic digital brain phantom. IEEE Transactions on Medical Imaging, 17(3):463-468, 1998.

[3] D. Donoho and I. Johnstone. Ideal spatial adaptation via wavelet shrinkage. Biometrika, 81:425-455, December 1994.

[4] S. Geman and D. Geman. Stochastic relaxation, gibbs distributions, and the bayesian restoration of images. In IEEE PAMI, volume 6, pages 721-741, 1984.

[5] G. Georgiou, S. Cohen, C. Piccoli, F. Forsberg, and B. Goldberg. Tissue characterization using the continuous wavelet transform part 1: decomposition method. IEEE trans. on ultras., ferro., and freq. cont., 48(2):355-363, 2001.

[6] M. Green. Statistics of images, the TV algorithm of Rudin-Osher-Fatemi. Technical report, Institute for pure and applied mathematics, UCLA, October 2002.

[7] S. Mallat. A theory for multiresolution signal decomposition: the wavelet representation. IEEE PAMI , 11(7):674-693, 1989. 
[8] Pizurica. Image denoising using wavelets and spatial context modeling. PhD thesis, Universite Gent, 2002.

[9] L. Rudin, S. Osher, and E. Fatemi. Nonlinear total variation based noise removal algorithms. Physica D, 60:259-268, 1992.

[10] J.L. Starck. Multiresolution support applied to image filtering and restoration. Graphical Models and Image Processing, 57:420-431, 1995.

[11] G. Winkler. Image analysis, random fields and dynamic Monte Carlo methods. Springer, 1995. 